\title{
Maxillofacial Fractures in Elderly Patients
}

Hiroaki Takakura $^{1,2}$, Tsuyoshi Shimo ${ }^{{ }^{*}}$, Norie Yoshioka ${ }^{1}$, Mayumi Yao ${ }^{1}$, Kyoichi Obata ${ }^{1}$, Soichiro Ibaragi ${ }^{1}$, Tatsuo Okui ${ }^{1}$, Yuki Kunisada $^{1}$, Ayaka Morisawa ${ }^{1}$, Akane Shibata ${ }^{1}$, Shoko Yoshida ${ }^{1}$, Yurika Murase ${ }^{1}$, Koji Kishimoto ${ }^{1}$, Akiyoshi Nishiyama $^{1}$, Hiroshi Mese ${ }^{2}$ and Akira Sasaki ${ }^{1}$

${ }^{1}$ Department of Oral and Maxillofacial Surgery, Okayama University Graduate School of Medicine, Dentistry and Pharmaceutical Sciences, Okayama, 700-8525, Japan

${ }^{2}$ Department of Dentistry and Oral Surgery, Fukuyama City hospital, Hiroshima, 721-8511, Japan

\section{Abstract}

Publication History:

Received: September 06, 2017

Background: According to the report of Japan's Ministry of Health, Labor and Welfare, looking at the Accepted: November 28, 2017 main reasons requiring nursing care by age group, elderly people aged 75 and over, falls and fractures Published: November 30, 2017 increase. The purpose of this study was to investigate the trends and characteristic features of maxillofacial fractures in over 75 years old, late stage of elderly patients.

\section{Keywords:}

Patients and Methods: Records of patients who were treated for maxillofacial fractures at the Oral and Maxillofacial fractures, Elderly, Maxillofacial Surgery department (Biopathology) of Okayama University hospital from January 2008 to Mandibular fossa, Maxillofacial December 2013 were retrospectively analyzed. Clinical records were analyzed in terms of age, gender, Surgery etiology, relevant medical history, and anatomical site of fracture. Patients $(\mathrm{n}=103)$ were divided into two groups by age: $<75$ years and elderly ( 75 years and older).

Results: Falls were responsible for the majority of fractures in the elderly group (81\%). The condylar process was the most common fracture site (53.8\%), among which cases $85.7 \%$ were accompanied with dislocation. The depth of the mandibular fossa was significantly shallower in the elderly than in young (age 10-29 years) patients.

Conclusion: In planning treatment, it is important to understand the characteristics and disease state of maxillofacial fractures of the elderly.

\section{Introduction}

With increasing life expectancy, the number of elderly receiving treatment for maxillofacial region fractures will increase [1]. According to the report of Japan's Ministry of Health, Labor and Welfare, the number of people aged 65 and older in the country will reach 36.57 million in 2025, with an expected peak of 38.78 million in 2042 , the percentage of people aged 75 and over will likewise increase, and is expected to exceed $25 \%$ in the year 2055 , looking at the main reasons requiring nursing care by age group, elderly people aged 75 and over, falls and fractures increase [2]. Trauma has a greater physical impact on elderly people because of their decreased physical reserves and age-related coexisting conditions including osteoporosis, reduced muscle mass, and cognitive decline [3]. Furthermore, since the number of lost teeth is greater among the elderly than in young people, management is complicated by difficult recovery of occlusal function and basic diseases [4]. Therefore, it is important to understand the characteristics and actual condition of maxillofacial fractures of the elderly in developing a treatment plan. In this study, we compile and analyze results from clinical examinations of maxillofacial fractures of elderly patients aged 75 years and older who underwent treatment in our department.

\section{Patients and Methods}

This was a retrospective study of all maxillofacial fractures seen at the Oral and Maxillofacial Surgery department (Biopathology) of Okayama University hospital. Clinical records from the 6-year period from January 2008 through December 2013 were analyzed in terms of age, gender, etiology, relevant medical history, and anatomical site of fracture. A total 103 patients were divided into two groups: $<75$ years and $\geq 75$ years ("elderly"). Fracture sites were identified as the condylar process, mandibular bone, maxillary bone, zygoma, coronoid process and alveolar bone. The condyle fracture was further defined according to the Lindahl classification into three subregions: the condylar head, condylar neck, and subcondylar process. The condyle fracture was also classified as no displacement, deviation displacement and dislocation [5]. The depth of the mandibular fossa was determined from the sagittal plane panoramic $x$-ray image by drawing a perpendicular line from the deepest part of the mandible fossa on the tangent to the highest point of the articular tubercle and measuring its length. We analyzed the data using the unpaired Student's t-test or a Chi-squared test of two groups for comparisons. Results are expressed as means \pm standard deviations (SDs). $P$-values $<0.05$ were considered significant.

\section{Results}

During the 6-year study period from January 2008 to December 2013, our department treated 103 patients for maxillofacial fractures, including $16(15.5 \%)$ aged 75 years or older. The ages of the elderly patients ranged from 75 to 90 years old with an average age of 79.8 years. Sex distribution was 9 males (56.3\%), 7 females (43.8\%), with a ratio of 1.2:1. Males of 75-79 years comprised the largest subgroup of the elderly fracture patients (Figure 1). The average number of days to visit was 4.81 days. The age range with the largest number of fractures was "Corresponding Author: Dr. Tsuyoshi Shimo, Department of Oral and Maxillofacial Surgery, Okayama University Graduate School of Medicine, Dentistry, and Pharmaceutical Sciences, 2-5-1 Shikata-cho, Kita-ku, Okayama 700-8525, Japan, Tel: +81-86-235-6702, Fax: +81-86-235-6704; Email: shimotsu@md.okayama-u.ac.jp

Citation: Takakura H, Shimo T, Yoshioka N, Yao M, Obata K (2017) Maxillofacial Fractures in Elderly Patients. Int J Surg Surgical Porced 2: 128. https://doi. org/10.15344/2456-4443/2017/128

Copyright: (c) 2017 Takakura et al. This is an open-access article distributed under the terms of the Creative Commons Attribution License, which permits unrestricted use, distribution, and reproduction in any medium, provided the original author and source are credited. 


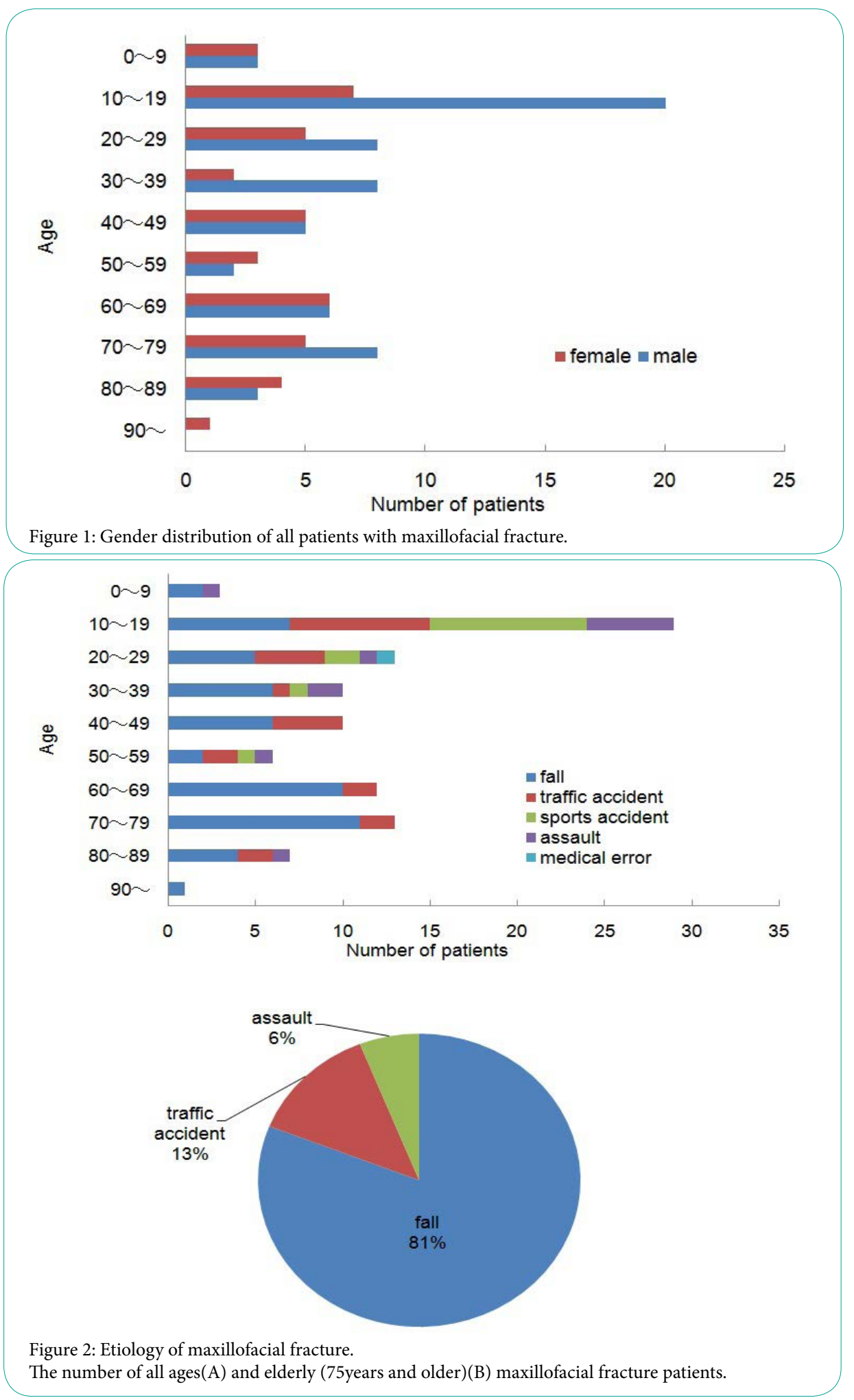

10-19 years, and the most common cause of maxillofacial fracture among this group was sports accidents $(31.0 \%)$ followed by traffic accidents (27.6\%), falls (24.1\%), and assaults (17.2\%) (Figure 2A). On the other hand, the most common cause of fracture in patients over 60 years was falls $(60-69$ years, $83.3 \%$; $70-79$ years, $84.6 \%$; $80-89$ years, $57.1 \%$ ) (Figure $2 \mathrm{~A}$ ); indeed, falls caused $81 \%$ of the fractures in the elderly group ( $\geq 75$ years) in this study (Figure $2 \mathrm{~B}$ ).
A significant medical history was noted in $100 \%$ of the elderly fracture patients, including hypertension (18.8\%), digestive system disease $(15.6 \%)$, diabetes $(9.4 \%)$, dementia $(9.4 \%)$, cardiovascular disease (12.5\%), hyperlipidemia (6.3\%), brain disease $(6.3 \%)$, and malignant neoplasm (6.3\%) (Figure 3). In terms of total number of fracture sites, patients under 75 years of age were affected most in the mandibular body $(29.0 \%)$, followed by the alveolar bone $(25.0 \%)$, 


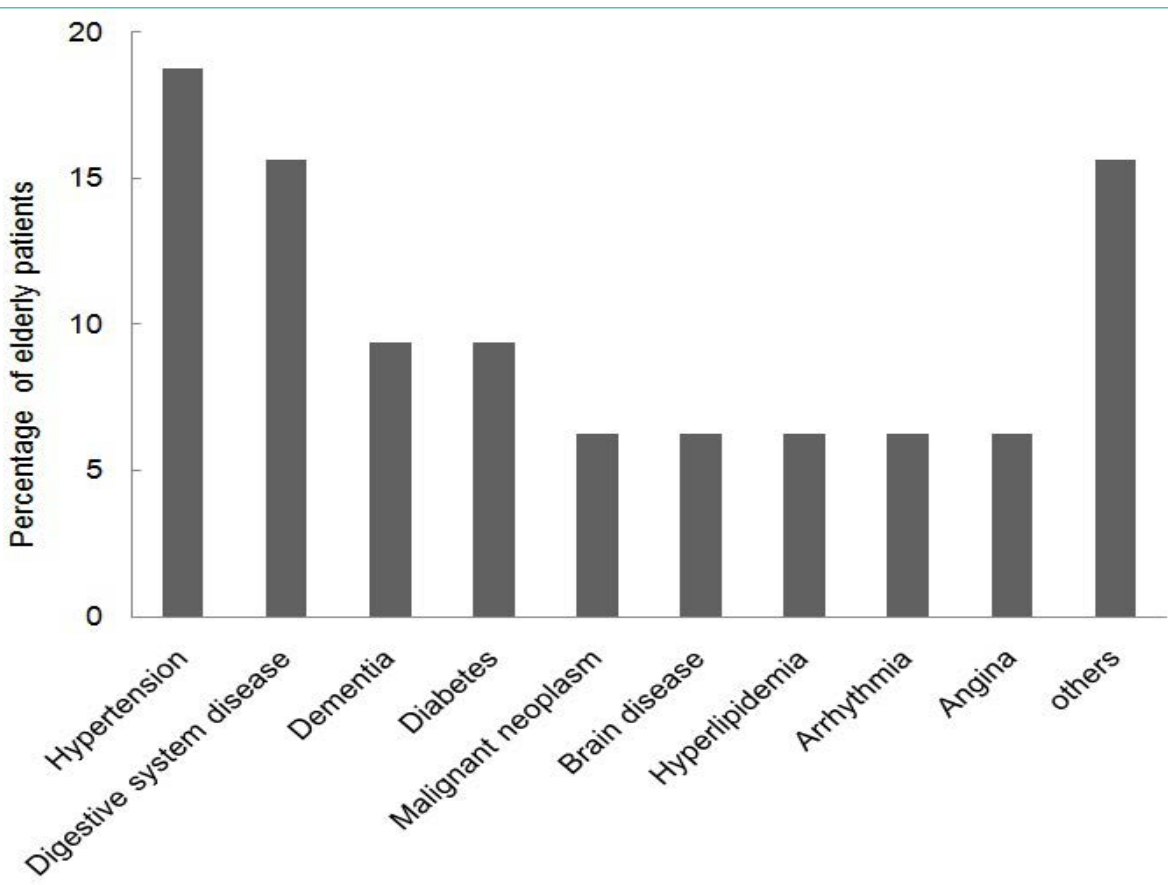

Figure 3: Number of patients with preexisting medical problems in elderly fracture patients (75 years and older).

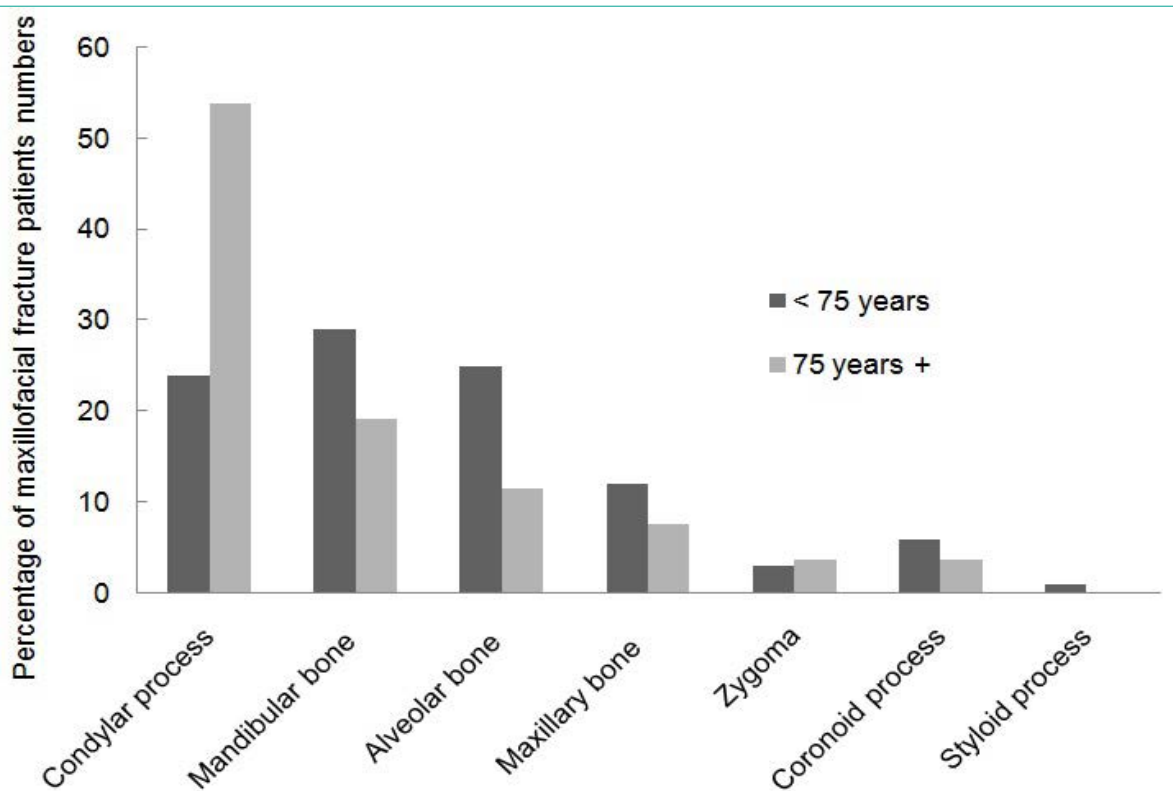

Figure 4: Site distribution of maxillofacial fracture: percentage of maxillofacial fractures at different sites among patients $<75$ years (white column) and $\geq 75$ years (black column).

condylar process $(24.0 \%)$ and maxillary bone (12.0\%). On the other hand, the condylar process $(53.8 \%)$ was the most common fracture site of patients over 75 years old $(\mathrm{p}<0.05)$, followed by the mandibular body (19.2\%), alveolar bone (11.5\%), and maxillary bone $(7.7 \%)$ (Figure 4).

For condylar process fracture site, according to Lindahl classification, patients under the age of $75(\mathrm{n}=78)$ included 20 cases (25.6\%) to the condylar head, 45 cases $(57.7 \%)$ to the condylar neck and 13 cases $(16.7 \%)$ to the subcondylar region, while in the patients $\geq 75$ years $(\mathrm{n}=14)$, there were 8 cases to the head $(57.1 \%), 4$ to the neck (28.6\%) and 2 to the subcondylar region (14.3\%) (Table 1).
In terms of the McLennan classification of condylar process fractures, patients under 75 years $(n=78)$ included 7 cases with no displacement (9.0\%), 15 with deviation (19.2\%), 14 with displacement (18.0\%), and 42 with dislocation (53.8\%), whereas patients aged 75 years or older $(\mathrm{n}=14)$ included 2 with no displacement $(14.3 \%)$ and 12 with dislocation (85.7\%) (Table 1). Dislocation cases in both age groups were most frequently accompanied by fracture of the head or neck. Therefore, we examined the relationship between the depth of the mandibular fossa and age in the condylar process fracture cases. The mandibular fossa depth was measured from the panoramic X-ray photograph (Figure 5A) and the depth was compared between young (10-29 years) and elderly patients was significantly shallower in the elderly than in the young patients $(\mathrm{p}<0.05)$ (Figure $5 \mathrm{~B})$. 
Citation: Takakura H, Shimo T, Yoshioka N, Yao M, Obata K (2017) Maxillofacial Fractures in Elderly Patients. Int J Surg Surgical Porced 2: 128. https://doi. org/10.15344/2456-4443/2017/128

Page 4 of 5

\begin{tabular}{|l|c|c|c|c|c|c|c|c|c|c|c|}
\hline \multicolumn{10}{l|}{ Maclennan classification } \\
\hline \multicolumn{3}{|l|}{ No displacement } & \multicolumn{2}{l|}{ Deviation } & \multicolumn{2}{l|}{ Displacement } & \multicolumn{2}{l|}{ Dislocation } & \multicolumn{2}{l|}{ total } \\
\hline & $<75 \mathrm{y}$ & $75 \mathrm{y}+$ & $<75 \mathrm{y}$ & $75 \mathrm{y}+$ & $<75 \mathrm{y}$ & $75 \mathrm{y}+$ & $<75 \mathrm{y}$ & $75 \mathrm{y}+$ & $<75 \mathrm{y}$ & $75 \mathrm{y}+$ \\
\hline
\end{tabular}

Table 1: Site distribution and the category of condylar process fracture.
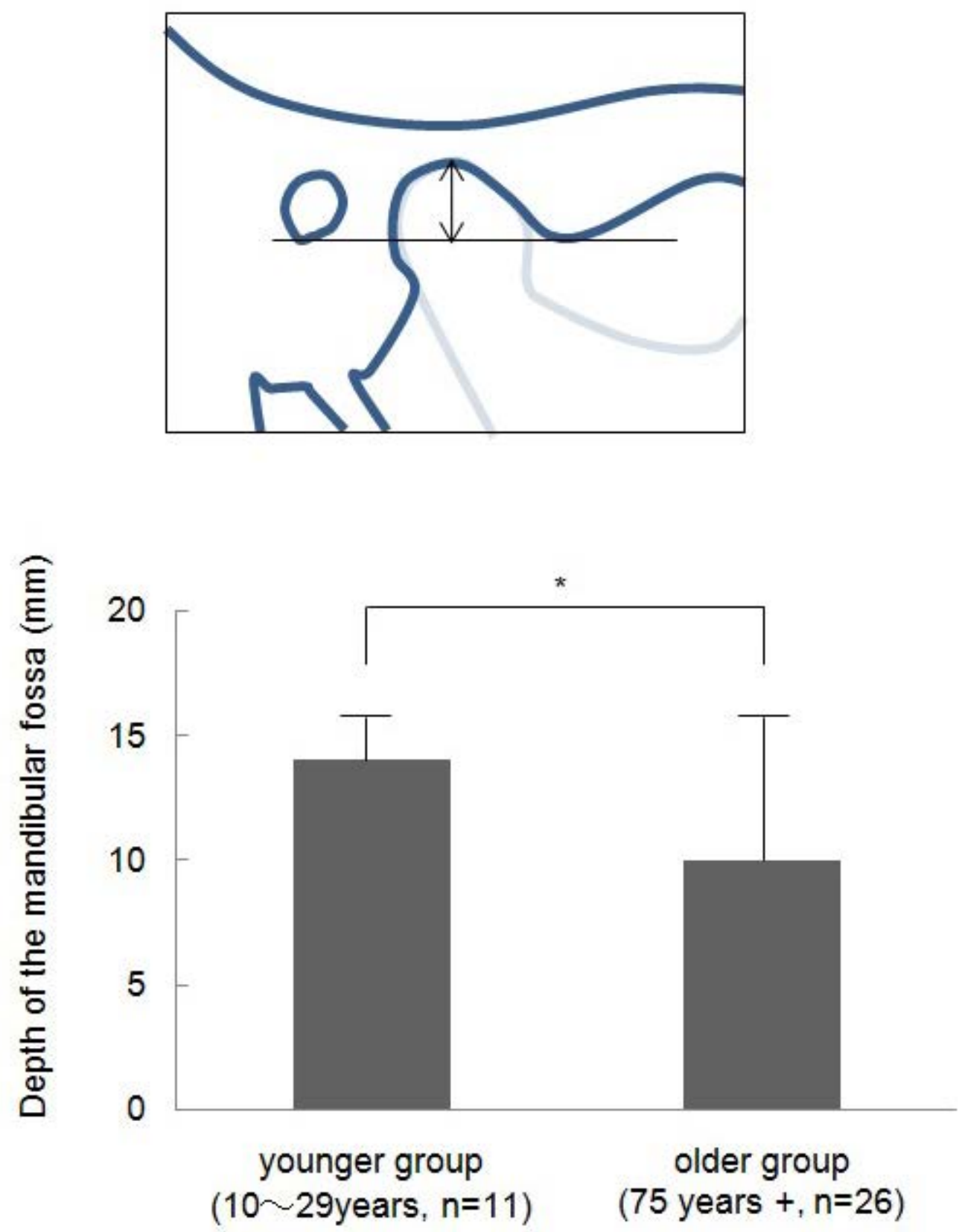

Figure 5: Comparison of the depth of the mandibular fossa between younger and older group.

A: The depth of the mandibular fossa was determined from panoramic x-ray image as described in Patient and Methods. B: Comparison of the depth of the mandibular fossa between younger (10-29 years) and older group ( 75 years and older). 


\section{Discussion}

In past reports of mandibular fractures, males have been more prevalent than females in both young and adult patient groups [6]. In the present data set of maxillofacial fractures, the 10-19 age range had the largest number of both male and female patients. Male patients were 2.9 times more numerous than females in that group; as the age increased, the ratio of male and female became almost equal. In the fractures of the elderly, there were almost no gender differences. This was thought to be due to the lack of gender differences in social activities or behaviors in older age people.

The elderly have many underlying diseases, and dementia and cognitive impairment increase the risk of falls and traffic accidents. There is an annual incidence of around 60\% [7]. Other risk factors for falls include disturbances of balance and gait, and cardiovascular risk factors, and these factors have been shown to be associated with recurrent falls [8]. Cardiovascular disease, angina (6.3\%) and arrhythmia (6.3\%) patients often take anticoagulants such as warfarin; in them, particular attention should be paid to the possibility of chronic subdural hematoma [9].

Compared to patients under 75 years of age, patients over 75 years of age show $53.8 \%$ more likelihood of having condylar process fractures $(p<0.05)$. As for the reason that the dislocated condylar process is observed in $85.7 \%$ of elderly patients with the condylar process fracture, it is presumed that the depth of the mandibular fossa is significantly shallow, causing the condylar process fracture, accompanied by interstitial dislocation on both sides. Although not shown in the data, we examined the relationship between the residual index and the condylar process fracture site, but there is no significant difference between them. However, in the case of elderly condylar process fracture patients, an estimated $75 \%$ are bilateral fractures of the condylar process. When edentulous patients fall, the external force received on the lower jaw is not propagated but is absorbed by the facial skull through the condylar processes, and most of the impact is absorbed by the mandible itself, so that fracture more easily occurs. Intermaxillary fixation is impossible in fractures of edentulous and multi-tooth defective patients because it is difficult to ensure reliable reduction. For elderly patients, it is desirable to shorten the period of opening restriction in order to limit stress and the need for supportive care. Therefore, in the case of mandibular body fracture, open reduction is performed in all cases, but in the case of condylar process fracture, preservation therapy is selected in all cases, because the jaw bone morphology and occlusion can be recovered by rehabilitation of oral and jaw function using dentures.

In this study, to understand the characteristics and actual condition of maxillofacial fractures in elderly patients aged 75 years or older, we analyzed clinical data such as age, gender, underlying disease, etiology, fracture site and treatment contents.

\section{Competing Interests}

The authors declare that they have no competing interests.

\section{References}

1. Velayutham L, Sivanandarajasingam A, O'Meara C, Hyam D (2013) Elderly patients with maxillofacial trauma: the effect of anageing population on a maxillofacial unit's workload. Br J Oral Maxillofac Surg 51: 128-132.

2. National institute of population and social security research (2012) Population projection for japan: 2011 to 2060.
3. American College of Surgeons Committee on Trauma. (2008) Geriatric trauma. in: ATLS: student course manual, 8th edition. ACLS, Chicago. 247-257.

4. NadhanLS, Balakrishnan VER (2014) Management of the edentulous mandible review article. Biomed Pharmacol J7: 143-146.

5. Neff A, Cornelius CP, Rasse M, Torre DD, Audigé L (2014) The comprehensive AOCMF classification system: condylar process fractureslevel 3 tutorial, Craniomaxillofacial Trauma Reconstr 7: S044-S058.

6. Atilgan S, Erol B, Yaman F, Yilmaz N, Ucan MC (2010) Mandibular fractures: a comparative analysis between young and adult patients in the southeast region of Turkey. J Appl Oral Sci 18: 17-22.

7. Shaw FE, Bond J, Richardson DA, Dawson P, Steen IN, et al. (2003) Multifactorial intervention after a fall in older people with cognitive impairment and dementia presenting to the accident and emergency department: randomised controlled trial. BMJ 326: 73-75.

8. Vassallo M, Sharma JC, Allen SC (2002) Characteristics of single fallers and recurrent fallers among hospital in-patients. Gerontology 48: 147-150.

9. Asghar M, Adhiyaman V, Greenway MW, Bhowmick BK, Bates A (2002) Chronic subdural haematoma in the elderly-a North Wales experience. JR Soc Med 95: 290-292. 